\title{
Effectiveness of low-dose theophylline for the management of biomass-associated COPD (LODOT-BCOPD): study protocol for a randomized controlled trial
}

Trishul Siddharthan ${ }^{1,2^{*}}$ (D), Suzanne L. Pollard ${ }^{1,2}$, Peter Jackson ${ }^{3}$, Nicole M. Robertson ${ }^{1,2}$, Adaeze C. Wosu ${ }^{2,4}$, Nihaal Rahman ${ }^{1,2}$, Roma Padalkar ${ }^{1,2}$, Isaac Sekitoleko ${ }^{5}$, Esther Namazzi ${ }^{5}$, Patricia Alupo ${ }^{5}$, John R. Hurst ${ }^{6}$, Robert Kalyesubula ${ }^{7,8}$, David Dowdy ${ }^{4}$, Robert Wise ${ }^{1,2}$, Peter J. Barnes ${ }^{9}$, William Checkley ${ }^{1,2}$ and Bruce Kirenga ${ }^{5}$

\begin{abstract}
Background: COPD is a leading cause of death globally, with the majority of morbidity and mortality occurring in low- and middle-income country (LMIC) settings. While tobacco-smoke exposure is the most important risk factor for COPD in high-income settings, household air pollution from biomass smoke combustion is a leading risk factor for COPD in LMICs. Despite the high burden of biomass smoke-related COPD, few studies have evaluated the efficacy of pharmacotherapy in this context. Currently recommended inhaler-based therapy for COPD is neither available nor affordable in most resource-limited settings. Low-dose theophylline is an oral, once-a-day therapy, long used in high-income countries (HICs), which has been proposed for the management of COPD in LMICs in the absence of inhaled steroids and/or bronchodilators. The Low-dose Theophylline for the Management of BiomassAssociated COPD (LODOT-BCOPD) trial investigates the clinical efficacy and cost-effectiveness of low-dose theophylline for the management of biomass-related COPD in a low-income setting.

(Continued on next page)
\end{abstract}

\footnotetext{
* Correspondence: tsiddhar@miami.edu

'Division of Pulmonary and Critical Care, University of Miami, School of

Medicine, Johns Hopkins University, 1951 NW 7th Ave, Suite 2308, Miami, FL 33136, USA

${ }^{2}$ Center for Global Non-Communicable Diseases, Johns Hopkins University, Baltimore, USA

Full list of author information is available at the end of the article
}

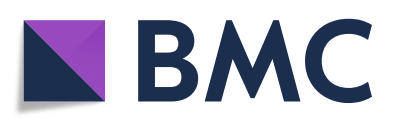

(- The Author(s). 2021 Open Access This article is licensed under a Creative Commons Attribution 4.0 International License, which permits use, sharing, adaptation, distribution and reproduction in any medium or format, as long as you give appropriate credit to the original author(s) and the source, provide a link to the Creative Commons licence, and indicate if changes were made. The images or other third party material in this article are included in the article's Creative Commons licence, unless indicated otherwise in a credit line to the material. If material is not included in the article's Creative Commons licence and your intended use is not permitted by statutory regulation or exceeds the permitted use, you will need to obtain permission directly from the copyright holder. To view a copy of this licence, visit http://creativecommons.org/licenses/by/4.0/. The Creative Commons Public Domain Dedication waiver (http://creativecommons.org/publicdomain/zero/1.0/) applies to the data made available in this article, unless otherwise stated in a credit line to the data. 


\begin{abstract}
(Continued from previous page)
Methods: LODOT-BCOPD is a randomized, double-blind, placebo-controlled trial to test the efficacy of low-dose theophylline in improving respiratory symptoms in 110 participants with moderate to severe COPD in Central Uganda. The inclusion criteria are as follows: (1) age 40 to 80 years, (2) full-time resident of the study area, (3) daily biomass exposure, (4) post-bronchodilator FEV $1 / F V C$ below the 5th percentile of the Global Lung Initiative mixed ethnic reference population, and (5) GOLD Grade B-D COPD. Participants will be randomly assigned to receive once daily lowdose theophylline (200 mg ER, Unicontin-E) or placebo for 52 weeks. All participants will receive education about selfmanagement of COPD and rescue salbutamol inhalers. We will measure health status using the St. George's Respiratory Questionnaire (SGRQ) and quality of life using the EuroQol-5D (EQ-5D) at baseline and every 6 months. In addition, we will assess household air pollution levels, serum inflammatory biomarkers (fibrinogen, hs-CRP), and theophylline levels at baseline, 1 month, and 6 months. The primary outcome is change in SGRQ score at 12 months. Lastly, we will assess the cost-effectiveness of the intervention by calculating quality-adjusted life years (QALYs) from the EQ-5D.
\end{abstract}

Trial registration: ClinicalTrials.gov NCT03984188. Registered on June 12, 2019

Trial acronym: Low-dose Theophylline for the Management of Biomass-Associated COPD (LODOT-BCOPD)

Keywords: Biomass, COPD, Theophylline

\section{Introduction}

Regular inhalation of toxins, such as tobacco smoke, can result in chronic obstructive pulmonary disease (COPD), a heterogeneous disease marked by largely irreversible airflow obstruction of the small airways, chronic bronchitis, and emphysema due to complex geneenvironment interactions over the lifetime [1,2]. COPD affects approximately 328 million people worldwide and is a leading cause of death globally [3]. The vast majority ( $90 \%)$ of morbidity and mortality occurs in low- and middle-income country (LMIC) settings. Although tobacco smoke is a leading risk factor for COPD globally, a significant proportion (20-30\%) of COPD occurs among never smokers $[4,5]$.

Household air pollution (HAP) from biomass combustion is an important risk factor in the development of COPD in many LMICs. Globally, nearly three billion people rely on biomass, which includes wood, dung, and agricultural crop waste or coal, for cooking and heating [6]. Individuals exposed to HAP in LMICs are $41 \%$ more likely to have COPD than those without the exposure [7]. Biomass-associated COPD has a distinct histopathology, phenotype and inflammatory profile when compared to tobacco-mediated COPD, suggesting a differential response to treatment and disease prognosis compared to tobacco-mediated disease $[1,8]$. Despite the high global burden of biomass-associated disease, little is known about the effectiveness of pharmacotherapies for biomassassociated COPD; to date, no clinical trials have focused specifically on treatment of biomass-associated COPD [8].

Theophylline has been used in the treatment of chronic obstructive airway diseases, including COPD and asthma, and remains widely prescribed worldwide, largely due to its low expense [9]. In many high-income countries, the frequency of side effects and the drug's narrow therapeutic index, together with wide availability of newer and more effective therapies, notably longacting bronchodilators, have led to reduced usage for management of COPD. However, a number of studies have demonstrated that theophylline at lower doses (1$5 \mathrm{mg} / \mathrm{L}$ ) results in improved respiratory symptoms via transcriptional downregulation of inflammatory genes [10-12]. Therapeutic monitoring is not necessary at such doses. Low-dose theophylline has been proposed as a treatment for biomass-associated COPD in LMICs, where inhaler-based therapy for COPD is usually unaffordable, not available or both $[9,13]$. Although no trials have been designed to evaluate the cost-effectiveness of treatment for COPD in LMICs, economic modeling demonstrates that annual per-capita costs for managing COPD with inhaler-based therapy would amount to USD 13,000-14,000 per disability adjusted life year (DALY) averted, well above cost-effectiveness benchmarks [14]. Previous studies among individuals with tobaccoassociated COPD have demonstrated low-dose theophylline monotherapy results in improved lung function $\left(\mathrm{FEV}_{1}\right)$, reduced respiratory symptoms, and decreased the frequency and duration of exacerbations [15].

The main objective of the Low-dose Theophylline for the Management of Biomass-Associated COPD (LODOTBCOPD) trial is to assess the clinical efficacy and costeffectiveness of low-dose theophylline for the management of biomass-associated COPD in a low-income setting.

\section{Design and methods \\ Study setting}

Uganda is a low-income country located in East Africa with a total population of 45 million and a million, over $80 \%$ of whom live in rural areas. The study will be carried out in Nakaseke, Central Uganda, a district covering 43,167 households with an estimated population of 208,500 . Nakaseke is located $14 \mathrm{~km}$ from the nearest 
highway and has been defined as rural by the Uganda Bureau of Statistics [16]. Most of the inhabitants (75\%) are subsistence farmers, and over $60 \%$ of them live on less than 45,000 shillings (\$12) per month [16]. The ratio of physicians and nurses per person are 1:25,000 and 1:5000, respectively, making Nakaseke one of the most underresourced health districts in Uganda. We have previously conducted a population-based study in Nakaseke using spirometry to assess the prevalence of COPD [17].

\section{Study population}

For this study, we will enroll adults with grade B-D COPD living in Nakaseke, previously identified in the GECo study [17, 18]. GECo was a population-based study which enrolled 3624 participants to validate casefinding instruments and assess the effectiveness of COPD self-management plan. The inclusion criteria are as follows: (1) age 40 to 80 years, (2) full-time resident of Nakaseke (lived in the area for $>3$ years), (3) daily biomass exposure, (4) post-bronchodilator $\mathrm{FEV}_{1} / \mathrm{FVC}$ below the lower limit of normal of the Global Lung Initiative Mixed Ethnic reference population [19, 20], and (5) GOLD Grade B-D COPD [2]. Exclusion criteria include the following: (1) plans to relocate within 1 year; (2) uncontrolled hypertension; (3) pregnancy; (4) current use of chronic respiratory medications (long-acting bronchodilators (LABA), long-acting muscarinic agents (LAMA), and inhaled corticosteroids (ISC); (5) pulmonary tuberculosis; (6) > 10 pack year tobacco smoking history and/or active smoking; and (7) known intolerance or contraindication to theophylline. All participants will additionally be COVID-19 tested by nasopharyngeal RT-qPCR $72 \mathrm{~h}$ prior to study visit.

\section{Study design}

We will enroll 110 adults in a randomized, double-blind, placebo-controlled trial to receive either daily $200 \mathrm{mg}$ ER low-dose theophylline ("intervention") or placebo ("control") (Fig. 1). The primary outcome of the trial will be to assess the clinical efficacy of low-dose theophylline at 12 months as determined by differences in health states as measured by the St. George's Respiratory Questionnaire (SGRQ). Secondary outcomes will include (a) differences in lung function decline and reversibility defined by ATS/ ERS [21] and (b) differences in health-related quality of life measured by the Euro-Qol 5D (EQ-5D). We will additionally evaluate the biologic activity of low-dose theophylline in subjects by measuring circulating inflammatory biomarkers and assess whether theophylline decreased inflammation. Finally, we will estimate the incremental cost-effectiveness of the intervention.

Participants in the intervention group will be provided with a monthly supply of low-dose theophylline tablets in childproof bottles by trained research assistants. Both arms will additionally receive COPD specific education and salbutamol inhalers per standard care [22]. All participants will be followed monthly for a period of 12 months.

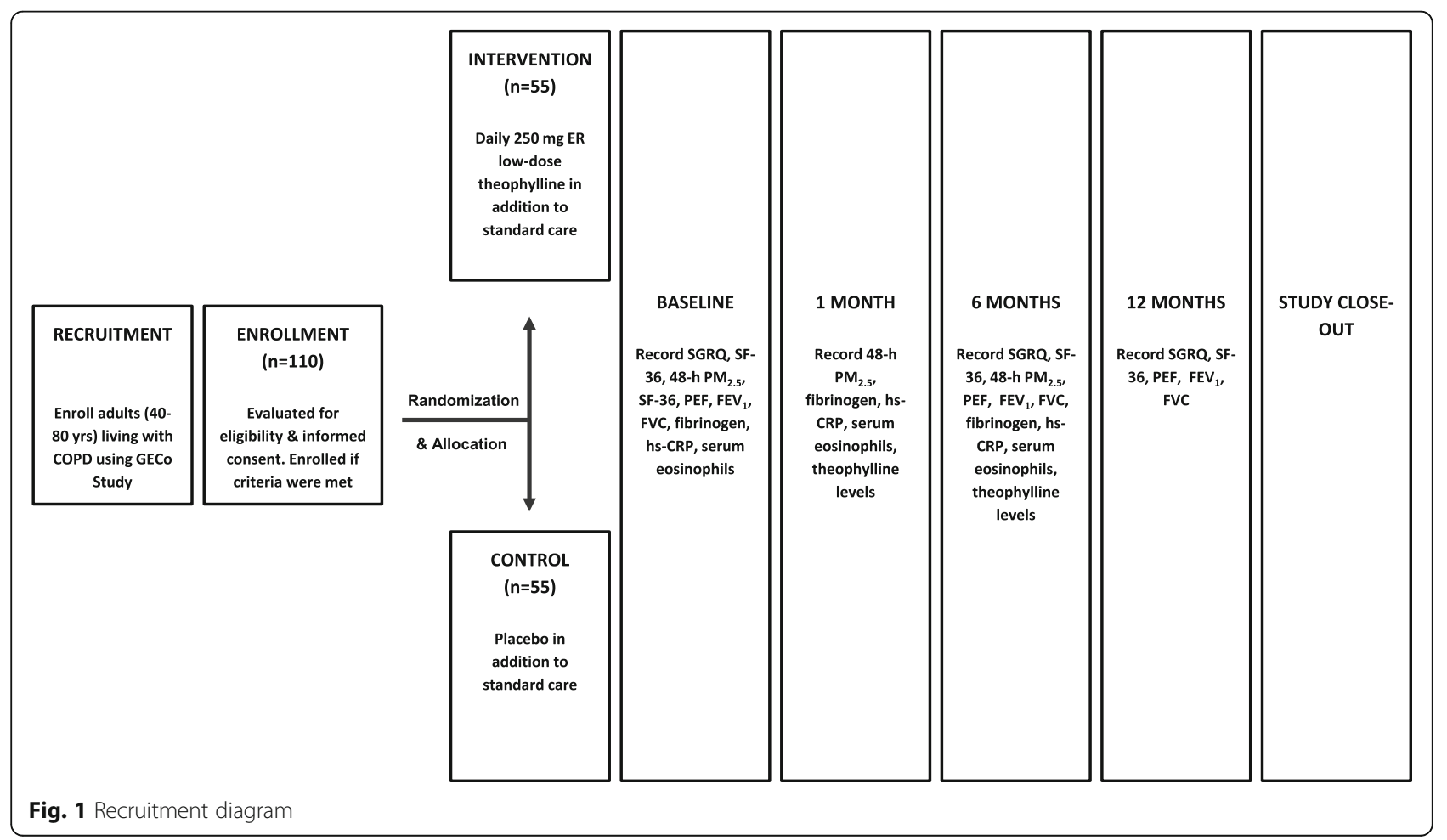




\section{Ethics}

The trial protocol was approved by the Institutional Review Boards (IRB) at Johns Hopkins School of Medicine (IRB 209008), the University of Miami (IRB 20201523), The Makerere College of Health Sciences School of Medicine (REF 2020-093), and the Uganda National Council of Science and Technology (HS 2758). The trial was also registered with ClinicalTrials.gov (Identifier: NCT03984188) on June 12, 2019. Prior to any data collection, research staff will explain study purpose and procedures to participants, emphasizing that participation is completely voluntary and participants can choose to withdraw from the study at any time. Participants will also be provided with an information sheet. Written consent will be obtained from each participant for the present study and future ancillary studies, if applicable. In situations where a participant is unable to read or write, a thumbprint will be obtained, along with written signature from a witness. Access to identifiable individual-level data will be restricted to an independent study clinician and trial pharmacist. Protocol amendments will be approved by all regulatory parties prior to change in research activities.

\section{Training}

All research personnel will receive human subjects training. Research assistants will be trained in electronic data capture and spirometry. Community health workers (CHWs) with experience in chronic respiratory diseases will provide COPD education [17]. CHWs have previously been educated on COPD pathophysiology, common treatments and their mechanism of action, as well as Ugandan guidelines for the diagnosis, management, and treatment of COPD, and familiarization with project goals.

\section{Recruitment, enrollment, and retention}

Individuals with previously identified COPD will be recruited from the GECo study [17]. GECo was a population-based study which screened 3634 participants for COPD and enrolled subjects into a selfmanagement trial. Trained research assistants will visit households to contact potential participants and invite them to the study. Before enrollment, study personnel will explain the goals of the study, what the study entails for the participant, and then ask if they are interested in participating. Those who agree to participate will be asked to complete detailed socioeconomic, medical history and exposure questionnaires and have spirometry performed for confirmatory testing.

\section{Randomization}

Once a participant is determined to be eligible and agrees to enter the study, research assistants will block randomize them to each of the two groups using the automated randomization feature in REDCap (Vanderbilt University Medical Center, Nashville, TN, USA). Participants will be followed monthly for a 1-year period, and enrollment will be staggered over a 1-year period. Principal investigators, members of the data coordinating center, and participants will be blinded to treatment allocation. Unblinding will occur only at the discretion of the data and safety monitoring board (DSMB) or at time of final analysis.

\section{Study arms}

Individuals randomized to the intervention arm will receive locally sourced low-dose theophylline $(200 \mathrm{mg}$ ER, Unicontin-E, Modi Mundi Pharma Pvt. Ltd.). Control randomized participants will receive methylcellulose placebo pills (Kampala Pharmaceutical Industries Ltd.) in identical packaging. The standard care for COPD as per WHO guidelines (salbutamol inhalers as needed) will be provided to all study participants, regardless of group assignment, by study clinicians. We will utilize standard dosing by ideal body weight (IBW). IBW is computed by using the Devine formulae: $\mathrm{IBW}_{\text {female }}=$ $45+0.9$ (height in $\mathrm{cm}-152$ ) $\mathrm{kg}$ and $\mathrm{IBW}_{\text {male }}=50+0.9$ (height in $\mathrm{cm}-152) \mathrm{kg}$ [23]. A dose of theophylline ER $200 \mathrm{mg}$ once daily (one placebo once daily) will be distributed to participants [24].

\section{Study outcomes}

We will follow-up participants at 1 month, 6 months, and 12 months during face-to-face assessments in a clinical setting (Figs. 1, 2, and 3). In the event that a participant is unable to attend a scheduled follow-up assessment visit because of an acute illness (e.g., exacerbation of COPD) or other reasons, the visit can be postponed, to within 2 weeks of the scheduled assessment visit. Research assistants will additionally perform monthly visits to assess medication adherence, refill medications, and assess for adverse events.

\section{COPD questionnaires}

The primary outcome of the trial will be a comparison of the change in SGRQ at 6 month increments from baseline to 12 months between the two arms [25]. The SGRQ measures impaired health and perceived wellbeing among individuals with chronic airway disease and offers many advantages for our study, namely (i) can be used to quantify changes in health following treatment, (ii) it is not limited to individuals with COPD, and (iii) it provides a standard metric that can be used for easy comparison across settings $[25,26]$. We have previously validated the SGRQ in Luganda, the most widely spoken language in Nakaseke [27]. We will additionally administer the COPD Assessment Test (CAT) at 6-month increments. The CAT measures the impact 


\begin{tabular}{|l|l|l|l|l|l|l|l|l|l|l|l|l|}
\hline Month & 2 & 4 & 6 & 8 & 10 & 12 & 14 & 16 & 18 & 20 & 22 & 24 \\
\hline Enrollment & & & & & & & & & & & & \\
\hline Recruit, consent, and enroll participants & & & & & & & & & & & & \\
\hline Follow up & & & & & & & & & & & & \\
\hline $\begin{array}{l}\text { Baseline (blood, spirometry, air pollution, EQ- } \\
\text { 5D, SGRQ) }\end{array}$ & & & & & & & & & & & \\
\hline 1-month (blood, spirometry, air pollution) & & & & & & & & & & \\
\hline 6-month (blood, spirometry, air pollution, EQ- & & & & & & & & & & & \\
$5 D$, SGRQ) & & & & & & & & & & & \\
\hline 12-month (spirometry, EQ-5D, SGRQ) & & & & & & & & & & & \\
\hline Dissemination and Analysis & & & & & & & & & & & \\
\hline
\end{tabular}

SGRQ: St. George Respiratory Questionnaire; EQ-5D EuroQol 5D;

Fig. 2 Schedule of enrollment, interventions and assessments

of COPD (cough, sputum, dyspnea, chest tightness) on health status [28].

\section{Quality of life}

The EuroQol-5D (EQ-5D) questionnaire will be administered at baseline and every 6 months through the trial period. The EQ-5D is a generic instrument for measuring health utility. It is based on a descriptive system that defines health in 5 dimensions: mobility, self-care, usual activities, pain/discomfort, and anxiety/depression [29]. Each dimension has 3 response categories corresponding to no problems, some problems, and extreme problems. The instrument is designed for self-completion, and respondents also rate their overall health on the day of the interview from 0 to 100 hash-marked, visual analog scale (EQ-VAS). Quality-adjusted life years (QALYs) will be derived using health utilities as estimated from the EQ5D. The EQ-5D has been widely tested and used in both general populations and patient samples and has been locally validated in Uganda [29].

\section{Lung function}

Spirometry will be conducted on all participants before and after bronchodilator therapy (400 mcg of salbutamol using a spacer) following standardized guidelines [19]. We will use the Easy on-PC handheld spirometer (ndd, Zurich, Switzerland), a device that has been validated and used in several large population-based studies [30, 31]. We will record post-bronchodilator PEF, $\mathrm{FEV}_{1}$, and FVC.

\section{Biomarkers}

We will assess fibrinogen levels, a biomarker for allcause mortality and exacerbations among those with COPD [32-34]. We will additionally measure serum hsCRP and blood eosinophils. We will conduct blood draws at baseline, 6 months, and 12 months with the

\begin{tabular}{|l|l|l|l|l|l|l|l|l|l|l|l|l|l|}
\hline Month & 0 & 1 & 2 & 3 & 4 & 5 & 6 & 7 & 8 & 9 & 10 & 11 & 12 \\
\hline Enrollment & & & & & & & & & & & & \\
\hline Recruit, consent, and enroll participants & & & & & & & & & & & & & \\
\hline Follow up & & & & & & & & & & & & & \\
\hline Spirometry & & & & & & & & & & & & \\
\hline Air pollution Measurements & & & & & & & & & & & \\
\hline Theophylline Levels & & & & & & & & & & \\
\hline Inflammatory Biomarkers & & & & & & & & & & \\
\hline Clinical Endpoints & & & & & & & & \\
\hline $\begin{array}{l}\text { Medication Refill, Assessment of Adverse Events and } \\
\text { Medication Adherence Survey }\end{array}$
\end{tabular}

Fig. 3 Schedule of enrollment, interventions, and assessments for participants 
aim of assessing response to theophylline as well as identifying sub-groups which may have a differential response to therapy. We will process and store serum samples in Uganda at $-80^{\circ} \mathrm{C}$ for future analysis. Samples will be destroyed at the request of participants.

\section{COPD exacerbations}

We will utilize ATS/ERS guidelines regarding exacerbation definition and severity. A COPD exacerbation will be defined as a worsening of patient's dyspnea, cough, or sputum beyond day-to-day variability [35]. Exacerbations will be treated with antibiotics and/or oral corticosteroids by study clinicians per standard protocol in both arms. A minimum of 2 weeks between consecutive exacerbations/hospitalizations will be used to consider events as separate in follow-up analysis [24].

\section{Health-care utilization}

Data on participant health care utilization will be collected. We will additionally collect data on concomitant medications/studies, outpatient visits, and any-cause hospitalizations during the previous month.

\section{HAP measurements}

We will measure personal $\mathrm{PM}_{2.5}$ concentrations using the UPAS (Access Sensor Technologies, Fort Collins, $\mathrm{CO})$, a gravimetric and real-time sampler. We will additionally collect continuous $\mathrm{PM}_{2.5}$ data using the OPC-N3 (Alpha Sense, Essex, UK). Participants will be encouraged to wear the monitors continuously during the 48-h period and to keep close while sleeping. Black carbon content of each personal filter will be determined using a validated optical attenuation measure [36, 37].

\section{Medication adherence}

We will measure adherence to prescribed medications at baseline and during follow-up visits. First, we will use the Adherence to Refills and Medications Scale-7 (ARMS-7) [38]. ARMS-7 will be administered at baseline and monthly during the follow-up period. The score is calculated by summing the scores for all items; lower scores indicate better adherence. Second, we will collect empty drug bottles and unused medication; compliance will be assessed by pill count [39]. Third, we will measure inhaler use by counters placed on salbutamol.

\section{Statistical analysis plan}

The primary study outcome will be to assess whether the low-dose theophylline intervention results in improved mean self-reported respiratory symptoms (SGRQ) compared to standard care at 12 months. For repeated outcome measurements (e.g., SGRQ, CAT, $\mathrm{PEF}, \mathrm{FEV}_{1}, \mathrm{FVC}$, serum biomarkers), linear mixed effects models will be used to account for within-subject correlation. The main analysis will be by modified intention-to-treat (ITT) based on cases where the primary outcome is available and will therefore rely on an assumption that data is missing at random. We will describe the number (\%) with missing primary outcome, look at reasons for missing the outcome, and consider characteristics of the patients excluded from the ITT analysis. Multiple imputation for the primary analysis will be used if the missing data exceeds $10 \%$ of randomized patients and as a secondary analysis regardless of the level of missingness.

Exposures (e.g., PM 2.5) at each follow-up will be aggregated to represent chronic exposure over the study period, as determined by the health outcomes. Analyses will be stratified to assess consistency across communities (rural and peri-urban) and be combined to obtain an overall risk estimate. In the combined analysis, we will adjust for community-level confounders.

We will examine repeated measurements of SGRQ by treatment group and carry out exploratory analyses to consider effects of the intervention over time. The SGRQ has previously been shown to have a standard deviation of 19.5 points in a similar population and a minimal clinically important difference of 4 points (a previous study involving low-dose theophylline resulted in a 7.8 point difference between intervention and control) [15]. A sample of 99 participants with COPD total will be needed to produce an $80 \%$ two-sided confidence interval that excludes a 4-point difference in SGRQ under the scenario of a 7.8 point difference in means [40]. We anticipate recruitment of 110 participants to account for attrition (55 per arm).

We will additionally conduct exploratory analysis to compare the exposure-response relationship between HAP and FEV 1 between study arms to assess whether theophylline attenuates the association. For the exposure-response associations, analyses will be conducted within the intervention and the control groups separately, as well as in a combined analysis. Non-linear associations between exposure and health outcomes will be examined using generalized additive models and other spline-based approaches [41]. We will estimate whether theophylline modifies the effect of HAP on lung function and respiratory outcomes via a principal stratification approach [42-44].

For evaluation of cost effectiveness, we will utilize measurements of the EQ-5D at baseline and months 3 and 6 to convert scores into health utility estimates using validated conversion formulae [45]. The incremental number of QALYs gained, comparing intervention participants to controls, can then be calculated by measuring the longitudinal values of health utility over the intervention period in each arm. We will adapt existing costing surveys from the parent trial in Uganda to 
adopt a societal perspective and include costs to participants, specifically monitoring productivity losses and costs from illness, in addition to costs of the program and the costs to society. For purposes of the cost-effectiveness analysis, the effectiveness of the intervention will be estimated as the incremental number of QALYs gained, as estimated from change in EQ-5D.

Uncertainty in the inputs of the cost-effectiveness analysis will be explicitly incorporated into the costeffectiveness analysis using probabilistic methods. Uncertainty in incremental costs and health benefits of each strategy will be presented using a scatter plot (the cost-effectiveness plane), and the probability of each implementation strategy being considered cost-effective for a range of thresholds will be presented using a costeffectiveness acceptability curve, displaying the probability of the intervention being cost effective at various levels of willingness-to-pay.

There is no universal benchmark for cost-effectiveness. Therefore, we will benchmark the cost-effectiveness of the intervention against a range of established willingness-topay thresholds [46]. We will compare the ICER to the WHO standard of gross domestic product per capita per QALY gained [47]. We will additionally compare our ICER to published estimates of incremental costeffectiveness for other similar health interventions in Uganda. We will lastly compare the total cost of the intervention to the average annual household income among participants [46].

\section{Data management and quality assurance}

Questionnaire-based data will be collected using REDCap (REDCap, Vanderbilt University Medical Center, Nashville, TN, USA) on password-protected tablet computers (Galaxy Tab A 10.1, Samsung Electronics Co., Suwon, South Korea) by trained research assistants. To protect confidentiality, all participants will be assigned a unique identification code, which will allow data to be deidentified and stored without identifying information. Identifiable information will only be accessible to the independent study physician, trial pharmacist, and members of the DSMB. Due to the low risks associated with this intervention, there are no pre-specified stopping rules though we will conduct an interim analysis at 6 months. We will conduct monthly checks of the data to assess completeness and outliers (data manager, principal investigator).

\section{Adverse events}

In previous trials, there were no significant differences between low-dose theophylline and placebo group. The most frequent drug-related adverse effects were stomach discomfort, headache, insomnia, and palpitations. We will collect data on safety and tolerability of theophylline, placebo, and salbutamol inhalers monthly, as well as provide contact information for study clinicians. We will maintain a data safety and monitoring board (DSMB) with reporting of all serious adverse events within $24 \mathrm{~h}$ by a study clinician. We will utilize health monitoring infrastructure of the parent trial to adjudicate adverse events. Health centers will be identified based on participants' residence and patients will be referred and transported for health-related events. The DSMB will audit trial conduct and adverse events every 12 months. Provisions for ancillary and post-trial care will be provided through trial insurance. We will report all adverse events in follow-up publication.

\section{Role of funder}

This study is funded by the National Institutes of Health (NHLBI/NIH). Peer review of the original grant application contributed to the final study design. A representative of the funder may attend DSMB meetings, though the funder otherwise has no role in the conduct or analyses of the study.

\section{Dissemination and data sharing}

The study results will be submitted for publication in peer-review journals and presentation at international meetings. Authorship will be determined by International Committee of Medical Journal Editors guidelines. The results will be additionally provided to the Ugandan Ministry of Health to develop national guidelines. We will make limited, de-identified datasets available for reproduction of any published analysis.

\section{Discussion}

This article discusses the rationale, methods and protocols for the LODOT-BCOPD randomized controlled trial in Uganda. The overall goal of the trial is to evaluate clinical efficacy and cost-effectiveness of low-dose theophylline treatment for biomass-associated COPD in a rural community in Uganda, in the absence of longacting bronchodilators and/or inhaled corticosteroids.

This study will include a total of 110 patients randomized in a 1:1 ratio to low-dose theophylline or placebo. Data will be collected to evaluate health-status improvement with widely used and locally validated questionnaires. In addition, lung function and biomarker testing will provide objective data to compare the two groups. Finally, analysis will evaluate the cost-effectiveness for the therapy, which is paramount for a disease that disproportionately affects LMICs. This study will be conducted in Nakaseke, Uganda, by a local and multinational research group with extensive experience conducting research in the region. This local experience will assist in navigating economic and cultural barriers to enrollment and allow community buy-in. This clinical trial builds upon previous research and collaborations in 
LMIC settings [17]. This previous research created well defined cohorts of patients with biomass COPD and has laid the groundwork for community partnerships that will facilitate recruitment and trust in the trial interventions. Furthermore, previous work has built capacity in the region and educated Ugandan nurses, physicians, and CHWs in research design and implementation.

This study has the potential to change the way we understand and treat biomass-associated COPD. Currently, there are no treatments that have been formally studied for biomass-associated COPD. Furthermore, in LMICs, where this disease is prevalent, standard COPD treatments are not widely available and unaffordable. This trial, if successful, could change the treatment strategy for millions of patients worldwide with this disorder and is scalable given the ease of administration and low cost.

\section{Trial status}

Enrollment for LODOT-BCOPD started on February 23rd 2021. Estimated date of completion is March 1 2023. Protocol version 1.4, March 1, 2021.

\section{Abbreviations}

LODOT-BCOPD: Low-dose Theophylline for the Management of BiomassAssociated COPD; COPD: Chronic obstructive pulmonary disease; LMICs: Lowand middle-income countries; HAP: Household air pollution; DALY: Disabilityadjusted life year; $\mathrm{FEV}_{1}$ : Forced expiratory volume; GECo: Global excellence in COPD outcomes; FVC: Forced vital capacity; ER: Extended release; IRB: Institutional Review Board; CHWs: Community health workers; IBW: Ideal body weight; SGRQ: St. George's Respiratory Questionnaire; EQ-5D: EuroQol5D; CAT: COPD Assessment Test; QALYs: Quality-adjusted life years; PEF: Peak expiratory flow; hs-CRP: High-sensitivity C-reactive protein; ATS: American Thoracic Society; ERS: European Respiratory Society; PM: Particulate matter; ARMS-7: Adherence to Refills and Medications Scale-7; ITT: Intention to treat

\section{Acknowledgements}

We acknowledge Crystal North, Engi Attia, John Balmes, and Ciprian Crainiceanu (Data Safety Monitoring Board); Richard Munana (Study Physician) and Isaac Segawa (Trial Pharmacist); and Adithya Cattamanchi and Kirsten Koehler for guidance on implementation and exposure assessments.

\section{Authors' contributions}

Conception and design: TS, SP, DD, RW, WC, and BK: drafting of manuscript: TS, SP, PJ, and NR; critical revision: TS, SP, PJ, NR, ACW, NR, RP, IS, EN, PA, JRH, $\mathrm{DD}, \mathrm{RW}, \mathrm{PB}, \mathrm{WC}$, and BK; all authors read and approved the final manuscript.

\section{Funding}

This study was funded by a Mentored Career Development Award through the National Heart, Lung and Blood Institute of the National Institutes of Health (K23 HL146946/HL/NHLBI). The funding agency has no role in the design of the study and collection, analysis and interpretation of data, and in writing the manuscript.

\section{Availability of data and materials}

Not applicable.

\section{Declarations}

\section{Ethics approval and consent to participate}

The trial protocol was approved by the Institutional Review Boards (IRB) at Johns Hopkins School of Medicine (IRB 209008), The University of Miami (20201523), The Makerere College of Health Sciences School of Medicine (REF 2020-093), and the Uganda National Council of Science and Technology
(HS 2758). The trial was also registered with ClinicalTrials.gov (Identifier: NCT03984188) on June 12, 2019. All participants will provide written informed consent prior to enrollment in the trial.

\section{Consent for publication}

Not applicable

\section{Competing interests}

The authors declare they have no competing interests.

\section{Author details}

${ }^{1}$ Division of Pulmonary and Critical Care, University of Miami, School of Medicine, Johns Hopkins University, 1951 NW 7th Ave, Suite 2308, Miami, FL 33136, USA. ${ }^{2}$ Center for Global Non-Communicable Diseases, Johns Hopkins University, Baltimore, USA. ${ }^{3}$ Division of Pulmonary and Critical Care, Virginia Commonwealth University, Richmond, USA. ${ }^{4}$ Department of Epidemiology, Bloomberg School of Public Health, Johns Hopkins University, Baltimore, USA. ${ }^{5}$ Makerere University Lung Institute, Makerere College of Health Sciences, Kampala, Uganda. ${ }^{6}$ UCL Respiratory, University College London, London, UK. ${ }^{7}$ Department of Physiology, Makerere College of Health Sciences, Kampala, Uganda. ${ }^{8}$ African Community Center for Social Sustainability, Nakaseke, Uganda. ${ }^{9}$ National Health and Lung Institute, Imperial College, London, UK.

Received: 2 September 2020 Accepted: 28 February 2021

Published online: 16 March 2021

\section{References}

1. Siddharthan T, Gupte A, Barnes PJ. COPD endotypes in low-and middleincome country settings: precision medicine for all. Am J Respir Crit Care Med. 2020:202(2):171-2.

2. Vogelmeier CF, Criner GJ, Martinez FJ, Anzueto A, Barnes PJ, Bourbeau J, Celli BR, Chen R, Decramer M, Fabbri LM. Global strategy for the diagnosis, management and prevention of chronic obstructive lung disease 2017 report. Respirology. 2017;22(3):575-601.

3. Alwan A. Global status report on noncommunicable diseases 2010. Geneva, Switzerland: World Health Organization; 2011.

4. Lamprecht B, McBurnie MA, Vollmer WM, Gudmundsson G, Welte T, Nizankowska-Mogilnicka E, Studnicka M, Bateman E, Anto JM, Burney P. COPD in never smokers: results from the population-based burden of obstructive lung disease study. CHEST Journal. 2011;139(4):752-63.

5. Tan W, Sin D, Bourbeau J, Hernandez P, Chapman K, Cowie R, FitzGerald J, Marciniuk D, Maltais F, Buist AS. Characteristics of COPD in never-smokers and ever-smokers in the general population: results from the CanCOLD study. Thorax. 2015;70(9):822-9.

6. Bonjour S, Adair-Rohani H, Wolf J, Bruce NG, Mehta S, Pruss-Ustun A, Lahiff M, Rehfuess EA, Mishra V, Smith KR. Solid fuel use for household cooking: country and regional estimates for 1980-2010. Environ Health Perspect. 2013;121(7):784-90. https://doi.org/10.1289/ehp.1205987. PubMed PMID: 23674502; PMCID: 3701999. Epub 2013/05/16

7. Siddharthan T, Grigsby MR, Goodman D, Chowdhury M, Rubenstein A, Irazola V, Gutierrez L, Miranda JJ, Bernabe-Ortiz A, Alam D. Association between household air pollution exposure and chronic obstructive pulmonary disease outcomes in 13 low-and middle-income country settings. Am J Respir Crit Care Med. 2018:197(5):611-20.

8. Pérez-Padilla R, Ramirez-Venegas A, Sansores-Martinez R. Clinical characteristics of patients with biomass smoke-associated COPD and chronic bronchitis, 2004-2014. Chronic Obstr Pulm Dis. 2014;1(1):23.

9. Barnes PJ. Theophylline in chronic obstructive pulmonary disease: new horizons. Proc Am Thorac Soc. 2005;2(4):334-9.

10. Cosio BG, Iglesias A, Rios A, Noguera A, Sala E, Ito K, Barnes PJ, Agusti A. Low-dose theophylline enhances the anti-inflammatory effects of steroids during exacerbations of COPD. Thorax. 2009;64(5):424-9.

11. Ford PA, Durham AL, Russell RE, Gordon F, Adcock IM, Barnes PJ. Treatment effects of low-dose theophylline combined with an inhaled corticosteroid in COPD. Chest. 2010;137(6):1338-44.

12. Barnes PJ. Theophylline. Am J Respir Crit Care Med. 2013;188(8):901-6.

13. Beran D, Zar HJ, Perrin C, Menezes AM, Burney P. Burden of asthma and chronic obstructive pulmonary disease and access to essential medicines in low-income and middle-income countries. Lancet Respir Med. 2015;3(2): $159-70$. 
14. Stanciole AE, Ortegón M, Chisholm D, Lauer JA. Cost effectiveness of strategies to combat chronic obstructive pulmonary disease and asthma in sub-Saharan Africa and South East Asia: mathematical modelling study. Bmj. 2012;344:e608

15. Zhou Y, Wang X, Zeng X, Qiu R, Xie J, Liu S, Zheng J, Zhong N, Ran P. Positive benefits of theophylline in a randomized, double-blind, parallelgroup, placebo-controlled study of low-dose, slow-release theophylline in the treatment of COPD for 1 year. Respirology. 2006;11(5):603-10.

16. Robertson NM, Nagourney EM, Pollard SL, Siddharthan T, Kalyesubula R, Surkan PJ, Hurst JR, Checkley W, Kirenga BJ. Urban-rural disparities in chronic obstructive pulmonary disease management and access in Uganda. Chronic Obstr Pulm Dis. 2019;6(1):17.

17. Siddharthan T, Pollard SL, Quaderi SA, Mirelman AJ, Cárdenas MK, Kirenga B, Rykiel NA, Miranda JJ, Shrestha L, Chandyo RK. Effectiveness-implementation of COPD case finding and self-management action plans in low-and middle-income countries: global excellence in COPD outcomes (GECo) study protocol. Trials. 2018;19(1):571

18. Vogelmeier CF, Criner GJ, Martinez FJ, Anzueto A, Barnes PJ, Bourbeau J, Celli BR, Chen R, Decramer M, Fabbri LM, Frith P, Halpin DM, López Varela MV, Nishimura M, Roche N, Rodriguez-Roisin R, Sin DD, Singh D, Stockley R, Vestbo J, Wedzicha JA, Agustí A. Global strategy for the diagnosis, management, and prevention of chronic obstructive lung disease 2017 report. GOLD executive summary. Am J Respir Crit Care Med. 2017;195(5):557-82.

19. Miller MR, Hankinson J, Brusasco V, Burgos F, Casaburi R, Coates A, Crapo R, Enright P, Van der Grinten C, Gustafsson P. Standardisation of spirometry. Eur Respir J. 2005;26(2):319-38.

20. Quanjer PH, Stanojevic S, Cole TJ, Baur X, Hall GL, Culver BH, Enright PL, Hankinson JL, Ip MS, Zheng J. Multi-ethnic reference values for spirometry for the 3-95-yr age range: the global lung function 2012 equations. Eur Respir J. 2012:40(6):1324-43.

21. Hankinson JL, Kawut SM, Shahar E, Smith LJ, Stukovsky KH, Barr RG. Performance of American Thoracic Society-recommended spirometry reference values in a multiethnic sample of adults: the multi-ethnic study of atherosclerosis (MESA) lung study. CHEST Journal. 2010;137(1):138-45.

22. Vallabhaneni K, Quaderi S, Flores O, Pollard S, Siddharthan T, Checkley W, Hurst J. Global excellence in COPD (GECo)-development of a COPD selfmanagement 'action plan' for low and middle income countries. Am Thoracic Soc Meet. 2019. p. A7105.

23. Shah B, Sucher K, Hollenbeck CB. Comparison of ideal body weight equations and published height-weight tables with body mass index tables for healthy adults in the United States. Nutr Clin Pract. 2006:21(3):312-9.

24. Devereux G, Cotton S, Barnes P, Briggs A, Burns G, Chaudhuri R, Chrystyn H, Davies L, De Soyza A, Fielding S. Use of low-dose oral theophylline as an adjunct to inhaled corticosteroids in preventing exacerbations of chronic obstructive pulmonary disease: study protocol for a randomised controlled trial. Trials. 2015;16(1):267

25. Jones PW, Quirk FH, Baveystock CM, Littlejohns P. A self-complete measure of health status for chronic airflow limitation: the St. George's Respiratory Questionnaire. Am Rev Respir Dis. 1992;145(6):1321-7.

26. Sherpa CT, LeClerq SL, Singh S, Pangeni R, Naithani N, Karki A, Chokhani RK, Han M, Gyetko M, Tielsch JM. Validation of the St. George's Respiratory Questionnaire in Nepal. Chronic Obstr Pulm Dis. 2015;2(4):281-9.

27. Morgan BW, Grigsby MR, Siddharthan T, Kalyesubula R, Wise RA, Hurst JR, Kirenga B, Checkley W. Validation of the Saint George's Respiratory Questionnaire in Uganda. BMJ Open Respir Res. 2018;5(1):e000276.

28. Jones P, Harding G, Berry P, Wiklund I, Chen W, Leidy NK. Development and first validation of the COPD Assessment Test. Eur Respir J. 2009:34(3):648-54

29. Brooks R. EuroQol: the current state of play. Health policy. 1996;37(1):53-72.

30. Jaganath D, Miranda JJ, Gilman RH, Wise RA, Diette GB, Miele CH, BernabeOrtiz A, Checkley W. Prevalence of chronic obstructive pulmonary disease and variation in risk factors across four geographically diverse resourcelimited settings in Peru. Respir Res. 2015;16(1):40.

31. Buist AS, McBurnie MA, Vollmer WM, Gillespie S, Burney P, Mannino DM, Menezes AM, Sullivan SD, Lee TA, Weiss KB. International variation in the prevalence of COPD (the BOLD Study): a population-based prevalence study. Lancet. 2007;370(9589):741-50.

32. Duvoix A, Dickens J, Haq I, Mannino D, Miller B, Tal-Singer R, Lomas DA. Blood fibrinogen as a biomarker of chronic obstructive pulmonary disease. Thorax. 2013;68(7):670-6.

33. Mannino DM, Tal-Singer R, Lomas DA, Vestbo J, Barr RG, Tetzlaff $K$, Lowings M, Rennard SI, Snyder J, Goldman M. Plasma fibrinogen as a biomarker for mortality and hospitalized exacerbations in people with COPD. Chronic Obstr Pulm Dis. 2015:2(1):23.

34. Mannino DM, Valvi D, Mullerova H, Tal-Singer R. Fibrinogen, COPD and mortality in a nationally representative US cohort. COPD: J Chron Obstruct Pulmon Dis. 2012;9(4):359-66.

35. Celli BR, MacNee W, Agusti A, Anzueto A, Berg B, Buist AS, Calverley PM, Chavannes N, Dillard T, Fahy B. Standards for the diagnosis and treatment of patients with COPD: a summary of the ATS/ERS position paper. Eur Respir J. 2004;23(6):932-46

36. Ahmed T, Dutkiewicz VA, Shareef A, Tuncel G, Tuncel S, Husain L. Measurement of black carbon (BC) by an optical method and a thermaloptical method: intercomparison for four sites. Atmos Environ. 2009;43(40): 6305-11.

37. Baumgartner J, Zhang Y, Schauer JJ, Huang W, Wang Y, Ezzati M. Highway proximity and black carbon from cookstoves as a risk factor for higher blood pressure in rural China. Proc Natl Acad Sci U S A. 2014;111(36):1322934. https://doi.org/10.1073/pnas.1317176111 PubMed PMID: 25157159; PMCI D: Pmc4246974. Epub 2014/08/27.

38. Kripalani S, Risser J, Gatti ME, Jacobson TA. Development and evaluation of the Adherence to Refills and Medications Scale (ARMS) among low-literacy patients with chronic disease. Value Health. 2009;12(1):118-23.

39. Farmer KC. Methods for measuring and monitoring medication regimen adherence in clinical trials and clinical practice. Clin Ther. 1999;21(6):1074-90.

40. Cocks K, Torgerson DJ. Sample size calculations for pilot randomized trials: a confidence interval approach. J Clin Epidemiol. 2013;66(2):197-201.

41. Hastie T, Tibshirani R. Generalized additive models for medical research. Stat Methods Med Res. 1995;4(3):187-96 Epub 1995/09/01. PubMed PMID: 8548102.

42. Hackstadt AJ, Matsui EC, Williams D, Diette GB, Breysse PN, Butz AM, Peng $\mathrm{RD}$. Inference for environmental intervention studies using principal stratification. Stat Med. 2014;33(28):4919-33.

43. Peng RD, Butz AM, Hackstadt AJ, Williams DAL, Diette GB, Breysse PN, Matsui EC. Estimating the health benefit of reducing indoor air pollution in a randomized environmental intervention. J Royal Stat Soc Ser. 2015;178(2): 425-43. https://doi.org/10.1111/rssa.12073.

44. Schwartz SL, Li F, Mealli F. A Bayesian semiparametric approach to intermediate variables in causal inference. J Am Stat Assoc. 2011;106(496): 1331-44.

45. Janssen MF, Pickard AS, Golicki D, Gudex C, Niewada M, Scalone L, Swinburn P, Busschbach J. Measurement properties of the EQ-5D-5L compared to the EQ-5D-3L across eight patient groups: a multi-country study. Qual Life Res . 2013;22(7):1717-27.

46. Revill P, Walker S, Madan J. Using cost-effectiveness thresholds to determine value for money in low- and middle- income country healthcare systems: are current international norms fit for purpose? York, UK: University of York; 2014.

47. GPD Per Capita: The World Bank; 2017 . Available from: https://data.worldba nk.org/indicator/NY.GDP.PCAP.CD. Accessed 1 Oct 2020.

\section{Publisher's Note}

Springer Nature remains neutral with regard to jurisdictional claims in published maps and institutional affiliations.

Ready to submit your research? Choose BMC and benefit from:

- fast, convenient online submission

- thorough peer review by experienced researchers in your field

- rapid publication on acceptance

- support for research data, including large and complex data types

- gold Open Access which fosters wider collaboration and increased citations

- maximum visibility for your research: over $100 \mathrm{M}$ website views per year

At BMC, research is always in progress.

Learn more biomedcentral.com/submission 